\title{
The Youth Mental Health Risk and Resilience Study (YouR-Study)
}

\author{
Peter J. Uhlhaas ${ }^{1 *}$, Ruchika Gajwani ${ }^{2}$, Joachim Gross ${ }^{1}$, Andrew I. Gumley², Stephen M. Lawrie ${ }^{3}$
} and Matthias Schwannauer ${ }^{4}$

\begin{abstract}
Background: The transition from adolescence to adulthood is associated with the emergence of psychosis and other mental health problems, highlighting the importance of this developmental period for the understanding of developing psychopathology and individual differences in risk and resilience. The Youth Mental Health Risk and Resilience Study (YouR-Study) aims to identify neurobiological mechanisms and predictors of psychosis-risk with a state-of-the-art neuroimaging approach (Magnetoencephalography, Magnetic Resonance Spectroscopy, Magnetic Resonance Imaging) in combination with core psychological processes, such as affect regulation and attachment, that have been implicated in the development and maintenance of severe mental health problems.

Methods/Design: One hundred participants meeting clinical high-risk criteria (CHR) for psychosis through the Comprehensive Assessment of At-Risk Mental State and Schizophrenia Proneness Instrument, Adult Version, in the age range from 16 to 35 years of age will be recruited. Mental-state monitoring up to a total of 2 years will be implemented to detect transition to psychosis. In addition, a sample of $n=40$ help-seeking participants will be recruited who do not meet CHR-criteria, a group of $n=50$ healthy control participants and a sample of $n=25$ patients with first-episode psychosis. MEG-activity will be obtained during auditory and visual tasks to examine neural oscillations and event-related fields. In addition, we will obtain estimates of GABA and Glutamate levels through Magnetic Resonance Spectroscopy (MRS) to examine relationships between neural synchrony and excitatory-inhibition (E/I) balance parameters. Neuroimaging will be complemented by detailed neuropsychological assessments as well as psychological measures investigating the impact of childhood abuse, attachment experiences and affect regulation.

Discussion: The YouR-study could potentially provide important insights into the neurobiological mechanisms that confer risk for psychosis as well as biomarkers for early diagnosis of severe mental health problems. Moreover, we expect novel data related to the contribution of affect regulation and attachment-processes in the development of mental health problems, leading to an integrative model of early stage psychosis and the factors underlying risk and resilience of emerging psychopathology.
\end{abstract}

Keywords: Adolescence, Psychosis, Early intervention, Biomarker, Affect regulation, Attachment

\section{Background}

The transition from adolescence to adulthood is associated with frequent mental health problems, ranging from affective disorders to more severe forms of psychopathology, such as psychosis [1]. Current data suggests that approximately one in every four to five youth will develop a mental disorder with severe impairment across

\footnotetext{
*Correspondence: peter.uhlhaas@glasgow.ac.uk

${ }^{1}$ Institute for Neuroscience and Psychology, University of Glasgow, 58

Hillhead Street, Glasgow, Scotland

Full list of author information is available at the end of the article
}

their lifetime [2]. Accordingly, understanding the developmental trajectory as well as potential ways to identify early signs of serious mental health problems is a key priority of current research [3].

Among mental health problems with an onset during the transition from adolescence to adulthood, psychotic disorders, such as those labelled as schizophrenia (ScZ), are a particular challenge given that certain cognitive and physiological signals are already present prior to the manifestation of psychosis [4]. Because the actual onset of psychotic symptoms is frequently accompanied by an 
extended prodromal phase of up to 5-6 years [5], clinical high-risk criteria (CHR) have been developed that allow the identification of young people at risk of developing psychoses. Firstly, Ultra-high risk (UHR) criteria are based on the presence of attenuated, psychotic symptoms as assessed by the Comprehensive Assessment of At-Risk Mental State (CAARMS) instrument [6] or the Structured Interview for Prodromal Symptoms (SIPS) [7]. Moreover, UHR-criteria on the CAARMS and SIPS instruments include a genetic risk and deterioration syndrome as well as brief limited intermittent psychotic episodes (BLIPs).

Secondly, CHR have been developed based on the basic symptom (BS) concept proposed by Huber and colleagues [8]. From this perspective, self-experienced perceptual and cognitive anomalies represent the earliest manifestation of psychosis risk while psychotic symptoms are considered as an adaptive response to render the anomalous cognitive experiences coherent [8]. Recent data from several studies using the Schizophrenia Proneness Instrument, Adult Version (SPI-A), have shown that CHR-criteria based on BS alone can identify young people at-risk for the development of psychosis with transition rates of up to $54.9 \%$ within four years period [9]. Similar transition rates have been originally observed for UHR-criteria, although recent studies have indicated that transition rates may be decreasing, ranging between 10 and 30\% [4]. Interestingly, the combination of both basic symptoms and UHR-criteria increase the predictive power [10].

While screening procedures are characterized by sufficient diagnostic accuracy to detect at-risk individuals [11], these approaches are currently not sensitive and specific enough to predict psychosis-risk on an individual level, a key objective for early intervention research. Accordingly, biomarkers may be required to boost prediction and furthermore to allow insights into the underlying neurobiology of the at-risk state. The search for underlying mechanisms und biomarkers is furthermore complicated by the fact that CHR-participants are a highly heterogeneous phenotype with genetic and environmental risk factors contributing to the at-risk state for psychosis, including trauma and affect dysregulation [12]. This is highlighted by recent findings that CHRparticipants who do not convert to psychosis develop a range of psychiatric disorders, including affective and personality disorders [13]. Accordingly, the CHR-state may not only confer risk for the development of psychosis per se but more broadly for diverse psychopathology [14].

\section{Rationale}

While data from neuroimaging and neurophysiology have demonstrated that many of the patterns observed in ScZ-patients are already present in at-risk individuals
[4], the underlying neurobiological and psychological mechanisms that give rise to subthreshold psychotic symptoms and self-experienced cognitive and perceptual disturbances remain poorly understood.

The search for biomarkers for early diagnosis and prognosis in CHR-participants has been largely conducted with anatomical and functional Magnetic Resonance Imaging (MRI/fMRI), brain imaging techniques with excellent spatial resolution. While useful in delineating the architecture of functional and anatomical networks, these techniques allow only indirect links to cellular and physiological mechanisms of circuit abnormalities [15]. Moreover, MRI approaches are unable to capture neural processes with high temporal resolution, which is essential for measuring fast rhythmic fluctuations of neuronal events that have been recently implicated in major psychiatric conditions [16].

Accordingly, the development of biomarkers may require a stronger focus on non-invasive techniques that allow a direct assessment of neuronal dynamics at high temporal resolution. This approach is supported by emerging evidence suggests that $\mathrm{ScZ}$ is associated with specific impairments in neural oscillations and their synchronization (neural synchrony), in particular at gamma-band frequencies $(30-200 \mathrm{~Hz})$ [15]. Brain oscillations have been shown to occur during normal brain functioning and are closely linked to the ability to perceive, memorize and attend to information [17-19]. Thus, it appears that brain oscillations could be a key to understanding the neurobiological mechanisms of psychosis-risk as well as provide biomarkers for predicting mental health outcomes, including psychosis, in CHR-individuals. Importantly, the impairments in neural synchrony are ideally suited for translational research because of evidence linking gamma-band oscillations during normal brain functioning to the integrity of GABAergic interneurons [20] and glutamatergic neurotransmission [21]. Supporting this hypothesis, the diagnosis of ScZ is associated with pronounced abnormalities in levels of GABA and Glutamate measured by magnetic resonance spectroscopy (MRS) [22]. Finally, blood and urine-samples will be collected to allow for potential genetic testing and proteomic analysis.

In addition to basic insights into the neurobiology of circuit dysfunction conferring risk for psychosis, the YouR-study will examine several core psychological processes involved in the maintenance and development of psychosis and several mental health problems, such as affectregulation and attachment. Evidence from behavioural and neuroimaging-studies suggests that problems in affect regulation may constitute a core risk factor in ScZ [23] which could be related to early trauma [24]. 
Preliminary evidence suggests that CHR-participants may experience greater difficulties in affect regulation [25] but the relationship to early trauma is unclear. Accordingly, we will further explore the role of affect regulation in at-risk participants and its relationship to psychosis as well as the role of attachment styles in the developmental trajectory of CHR-participants and its relationship to clinical variables.

\begin{abstract}
Aims
The primary aim of the YouR-study is to characterize changes in neural oscillations in CHR-participants and to develop a biomarker for psychosis-prediction and mental health outcomes. Accordingly, we will recruit a sample of $n=100$ CHR-individuals for psychosis and apply MEG during cognitive tasks and resting-state activity in combination with advanced analytic tools to identify the frequencies as well as brain regions involved in aberrant neural synchrony. In addition, to establish links between neural synchrony and E/I-balance parameters MRS-measured GABA/Glutamate levels in auditory and visual cortices will be obtained. During a follow-up period of up to two years, transition to psychosis, functional status and changes in affect-regulation will be monitored. Moreover, the study will identify the relationship between disturbances in affect regulation, attachment and trauma and their contribution towards the development of psychosis.
\end{abstract}

\section{Methods/Design}

The study will be a longitudinal cohort design with CHR-participants using neuroimaging to investigate brain activity and psychological measures to identify risk and resilience factors as well as biomarkers for emerging mental health problems. The YouR-Study will be performed according to the Research Governance Framework for Health and Community Care (Second edition, 2006).

\section{Recruitment and participants}

We aim to recruit up to $n=100$ participants meeting CHR-criteria in the age range from 16-35 years and we expect to identify from this participant group an additional $n=25$ participants who meet criteria for FEP over a four-year period. The recruitment of the CHR- and FEP-groups will involve NHS-patients services in NHS Greater Glasgow and Clyde and NHS Lothian, NHS First Episode Psychosis Services, Community Mental Health Teams (CMHTs), Primary Care Mental Health Teams (PCMHTs), Clinical Psychology Services, Community Adolescent Mental Health Services (CAMHS), and NonGovernmental mental health organisations. Recruitment for the YouR-study began in October 2014 and is expected to continue until 12/2017.
In addition, the YouR-study recruits from the general population through a website http://www.your-study. org.uk which includes an initial screening through the 16-item version of the prodromal questionnaire (PQ) [26] and a 12-item scale of perceptual and cognitive items. Previous data suggested that a cut-off score of 6 or more positively answered items on the 16-item version of the PQ produced correct classification of CHR-criteria based on CAARMS-interviews with high sensitivity and specificity [26].

CHR- and FEP-groups will be compared to two groups of participants: 1) A group of $n=40$ help-seeking participants below the threshold for UHR- or SPI-A criteria who will be recruited through the same recruitment pathways as participants meeting CHR-criteria (lowrisk group). The reason for the inclusion of this group is to control for non-specific factors associated with CHR-individuals, such as the presence of co-morbid non-psychotic psychopathology, drug abuse and lower educational status. And 2) A group of $n=50$ participants without the presence of any current DSM-IV disorder, current substance abuse and first-degree relative with psychosis.

\section{Clinical-assessments and CHR-criteria}

To establish CHR-criteria, the CAARMS-Interview [15, 16] and the COGDIS/COPER items for the SPI-A [27] are administered. Participants are recruited into the CHR-group if they meet a) SPI-A COGDIS/COPER-criteria b) ARMS attenuated psychosis group (subthreshold psychotic syndrome present in the last year without a decline in functioning) c) ARMS vulnerability group (family history of psychosis plus a 30\% drop in GAF) and d) ARMS BLIPs-group (brief limited intermittent psychotic symptoms). In addition, the M.I.N.I. International Neuropsychiatric Interview (M.I.N.I. 6.0) [28] will be administered to identify psychiatric comorbidity as well as the scales for premorbid adjustment [29] and social and functional role scale will be administered [30]. Previous studies indicated that these variables are significantly impaired in CHRparticipants and are predictors for transition to psychosis and functional outcome [31-33].

\section{Neuropsychology}

CHR-participants are characterized by cognitive deficits in several domains, including processing speed and verbal memory [34], which are predictors for transition to psychosis [35]. The neuropsychological assessment of the YouR-study consists of the Brief Assessment of Cognition in Schizophrenia Battery (BACS) which has been extensively used in ScZresearch [36]. In addition, the following tasks from the University of Pennsylvania Computerized 
Neuropsychological Testing Battery (PennCNP [37]: a) Continuous Performance Test b) N-Back Task and c) Emotion Identification Task will be included in the neuropsychological assessment. Finally, the Edinburgh Handedness Inventory [38] and the National Adult Reading Test [39] will be administered.

\section{Questionnaires}

Several psychological measures will be used in order to identify mechanisms of change and predictors of transition to psychosis and outcome. All are brief self-report scales, which have good psychometric properties. These include: 1) The Beliefs About Paranoia Scale (BAPS) [40] 2) The Brief Core Schema Scale (BCSS) [41] 3) The Psychosis Attachment Measure (PAM-SR) [42] 4) Adverse childhood experience scale (ACES) [43] and 5) The Rust Inventory of Schizotypal Cognitions (RISC) [44] 6) Inventory of interpersonal problems [45]- 32 item Version 7) The Significant Others Scale [46] and 8) The International Positive and Negative Affect Schedule, short-form (I-PANAS-SF) [47] and 9) Social Interaction Anxiety Scale [48] and the Functional Remission in General Schizophrenia (FROGS) scale [49].

\section{Follow-up}

Follow-up interviews will be conducted every 3-6 months with CHR and low-risk participants. This will include subscales of the CAARMS as well as questionnaires to examine stress-levels, interpersonal functioning and affect regulation. In addition, the SCID I interview and the social and functional role scales will be added at follow-up appointments at 6,12 and 24 months.

\section{Outcome parameters}

In addition to conversion to psychosis in CHRindividuals, the YouR-study will examine changes in global functioning as well as role and social functioning as important outcome parameters in CHRparticipants.

\section{Neuroimaging}

MEG

All neuroimaging experiments will be conducted within the facilities of the Centre for Cognitive Neuroimaging (CCNi), University of Glasgow. MEG-data will be recorded with a whole-head 248-channel 4D Neuroimaging WH3600 system at $1017.25 \mathrm{~Hz}$ sampling-rate and filtered with an online $400 \mathrm{~Hz}$ low-pass filter (DC$400 \mathrm{~Hz}$ ).

MEG signals will be pre-processed and analyzed using the open source matlab-toolbox fieldtrip [50] and customized scripts. Data-analyses will be conducted at sensor and source-level in the $1-150 \mathrm{~Hz}$ frequency range. Time-frequency representations will be estimated through Morlet-Wavelets and Multi-tapers. In addition, event-related fields (ERFs) during auditory mismatch negativity (MMNm) and sensory attenuation will be examined. MEG is ideally suited for the analysis of source-activity and source-reconstruction techniques, such as beamformers [51], and Minimum Norm Estimates (MNE) will be employed [52].

We will employ individual head models using a common dipole grid in MNI space. Cortical segmentation and surface reconstruction are performed using SPM8 as well as spatial alignment of structural MRIs. In a second step, we will select peak voxels for the reconstruction of virtual channels which will allow the examination of the time course of oscillatory activity within a specified frequency band and the analysis of functional connectivity with measures such as Partial Directed Coherence (PDC) [53], Granger Causality [54] and Phase-Lagged Synchronization [55].

\section{Experimental tasks}

Resting-state measurement: We will obtain resting-state data in an eyes-open condition (duration: $4 \mathrm{~min}$ ) which provides important evidence on the organization of large-scale networks and their impairments.

Visual Grating Task: We will employ a visual task that was designed to elicit robust high-frequency activity in the context of focused attention [56]. On each trial, participants are shown a circular sine wave grating at central screen fixation that contracts towards the fixation point. Participants are required to press the response button with their right index finger when the stimulus increases in velocity which occurs between 750 and $3000 \mathrm{~ms}$ post stimulus onset (10\% of the trials are catch trials in which no acceleration occurred). Extensive evidence suggests that high-frequency oscillations are robustly elicited by this paradigm with a signal-increase at the source-level of $\sim 200 \%$ and excellent test-retest reliability in controls measured with MEG-data [57]. Moreover, data in healthy controls [58] and invasive electrophysiological data [59] suggest tight correlations of gamma-band fluctuation with reaction times (RTs) RTs as well as with detection rates in ScZ-patients [60].

An auditory steady-state (ASS) paradigm: Kwon et al. [61] conducted the first $40 \mathrm{~Hz}$ ASSR-study in ScZpatients using EEG and reported reduced power and synchronization to $40 \mathrm{~Hz}$ stimulation. These initial findings have been replicated by several groups in EEG- and MEG-recordings (for a review see [62]). Participants are presented auditory stimuli consisting of 2000-msec $1000 \mathrm{~Hz}$ carrier tones, amplitude modulated at $40 \mathrm{~Hz}$ (100 trials). In addition to the passive presentation of ASS-stimuli, participants will be asked to initiate the same auditory stimuli through button press which allows 
for a comparison between auditory responses during a self-initiated sensory processing vs. passive stimulation or (sensory attenuation) [63]. Evidence suggests that both ScZ-patients as well as CHR-individuals are impaired in sensory attenuation [64].

Auditory MMNm Paradigm: MMN is an event-related potential/field elicited automatically by violations of previously established auditory regularities [65]. MMN amplitudes have been consistently found to be attenuated in both medicated and unmedicated ScZ- patients [66] Moreover, recent studies have found MMN signals to be present already in CHR-participants, indicating that MMN-deficits could represent a biomarker for psychosis development [67].

The YouR MEG-battery will investigate MMNm amplitude responses to both duration deviants and sound omissions during an auditory oddball paradigm in which three different sequences of auditory stimuli will be presented binaurally at $70 \mathrm{~dB}(150 \mathrm{~ms}$ SOA, $700-1000 \mathrm{~ms}$ ISI). In addition, an omission sequence which contains only four identical tones is included to examine auditory predictions. Participants are instructed to focus their attention away from the sounds and to perform a simple visual detection task.

\section{MRS-spectroscopy}

We will examine both GABA and Glutamate/Glutamine (Glx) levels with $1 \mathrm{H}$-MRS in three $2 \times 2 \times 2 \mathrm{~cm}^{3}$ singlevolume ROIs 1) right visual cortex and 2) left and right auditory cortex to examine correlations between fluctuations in neural oscillations GABA/Glutamate levels. 1HMRS spectra will be acquired on a $3 \mathrm{~T}$ Siemens Magnetom Tim Trio MRI Scanner, using a 32-channel radiofrequency head coil and a MEGA point-resolved spectroscopy (PRESS) spectral J-difference editing sequence. The following parameters will be used: TR/TE 1500/68 ms, $1200 \mathrm{~Hz}$ acquisition bandwidth, vector size of 512, 256 averages (128 editing-on and 128 editing-off scan) and a delta frequency of -1.7 (on scan; special symmetrical editing approach to remove contamination of co-edited macromolecules at $1.9 \mathrm{ppm}$ ) and $7.5 \mathrm{ppm}$ (off scan).

To assess glutamate levels, both physiologically active and inactive glutamate are measured (Glu). The proposed sequence (MEGA-PRESS) allows the detection of an aggregated signal representing the sum of glutamate and glutamine (Glx). Because the majority of physiologically active glutamate is derived from glutamine, high levels of Glx suggest elevated glutamatergic activity. We expect reduced GABA-levels but elevated Glx levels in the CHR-group which correlate with reduced power and peak-frequency of $\gamma$-band activity.

\section{Anatomical imaging: MRI/DTI}

T1 weighted anatomical data will be acquired using a 3D MPRAGE sequence $(\mathrm{FoV}=256 \times 256 \times 176 \mathrm{~mm} 3$, voxel size $=1 \times 1 \times 1 \mathrm{~mm} 3, \quad \mathrm{TR}=2250 \mathrm{~ms}, \mathrm{TE}=2.6 \mathrm{~ms}$, TI $=$ $\left.900 \mathrm{~ms}, \mathrm{FA}=9^{\circ}\right)$. For the analysis of MRI, we will focus on measures of cortical thickness as well as volume of gray and white matter. In addition to whole brain analyses, we will focus on ROIs in the auditory and visual cortex to correlate alterations in structural variables with MEG-parameters.

Moreover, the YouR-study will acquire DTI-data with the following sequence: 2D slice selective spin echo EPI sequence with diffusion encoding, 89 axial slices with a thickness of $1.7 \mathrm{~mm}, \mathrm{TR} / \mathrm{TE}=1200 \mathrm{~ms} / 100 \mathrm{~ms}$, bandwidth $=1346 \mathrm{~Hz} /$ pixel, echo spacing $=0.85 \mathrm{~ms}$. For DTI analysis, 67 diffusion encoding directions with a b-value of $1000 \mathrm{~s} / \mathrm{mm} 2$ will be covered.

\section{Sample size and power calculation}

Our current research with MEG has demonstrated large effect sizes for deficits in high-frequency oscillations in chronically medicated ScZ-patients as well as in medication-naïve FE-ScZ patients (chronic ScZ: $d=1.26$; FE-ScZ $d=1.0)$ [68, 69]. Because we will employ novel and more advanced analyses approaches for the proposed project, we are confident that we will maximise the possibility to detect dysfunctions in CHR-participants that will be in the range and above of effect sizes currently available for prodromal ScZ-research.

Previous studies with a variety of methods, such as MRI, fMRI as well as event-related potentials (ERPs), have demonstrated anatomical and physiological impairments in CHR-cohorts with medium to large effect sizes [70]. Electrophysiological parameters were among those deficits with the largest effects [67]. For example, Atkinson et al. [71] demonstrated an impairment in MMN in CHR-participants vs. controls of $d=.75$. Accordingly, given a sample of $n=50$ controls and 100 CHR-participants and an estimated effect size of .75 for the current study, the power to detect significant differences in MEG-parameters between controls and CHR-participants is $97 \%$.

In regards to the ability to distinguish between CHRparticipants who will transition to psychosis vs. CHRparticipants without conversions, previous published effect sizes have reported medium but also large effect sizes for differences on anatomical and functional parameters [70]. For the current study, a conservative, medium effect-size of $d=.50$ for a sample of $n=30$ converted CHR-participants vs. 70 CHR-non converted CHR-participants will yield a statistical power to detect significant differences between converted vs. nonconverted CHR-participants of $82 \%$. The sample of $n=$ 30 converted CHR-participants is consistent with a 
meta-analysis on conversion rates in CHR-participants over a two year period [4]. Should CHR-participants be lost in the follow-up period, we will recruit additional participants during the course of the project.

\section{Statistical analysis}

Interim analysis of neuroimaging and clinical data will be carried out after the recruitment of $n=50 \mathrm{CHR}$, 25 low-risk and 25 control participants. We will systematically explore relationships between MEGvariables (task and resting- state) and GABA/Glutamate levels with psychopathological variables (CAARMS, SPI-A) and neuropsychology (BACS) in the CHR-group. Specifically, we will identify those MEG (sensor, frequency and source-regions) and MRS-parameters with the largest effect size and perform information theoretical analysis to identify linear and non-linear dependencies.

In addition to parametric analysis of group differences in baseline-variables, we will employ a multivariate machine learning technique towards the development of predictors for conversions and functional outcome in CHR-individuals, an approach which has been used recently in the neuroanatomical classification of ScZ [72]. In the first step, we will reduce the dimensionality of the MEG-data by focusing on reconstructed source-time courses with significant task effects (baseline vs. task). For resting-state data, we will employ a principal component analysis (PCA) to the source-reconstructed activity in the $4-200 \mathrm{~Hz}$ frequency range in order to reduce the number of variables.

To examine transitions, we employ a Generalized Linear Model (GLM) to assign MEG-data of individual subjects to one of the three designated categories (controls vs. transitioned CHR vs. non-transitioned CHR). Following a 10-fold cross validation, we will divide the total sample into 10 non-overlapping samples. Each sample will be iteratively held back as test data while the classifier is trained on the 9 remaining samples. A multiclass logistic regression (GLM) ranks features by their estimated effect on the resulting model's performance. A general ranking scheme will combine the results obtained from 10 iterations. A subset of 3 to 10 top ranking signal features will be re-evaluated and the combination of features resulting in the best classification performance will serve as the diagnostic MEGindex.

\section{Discussions}

Evidence has accumulated that the onset of severe mental health problems in adolescence, such as psychosis, is preceded by alterations in functional and anatomical brain networks $[71,72]$ as well as with changes in psychological processes related to affect regulation [26].
Accordingly, further insights into the neurobiological as well as psychosocial factors that confer risk and resilience towards the development of psychosis and poor functional outcomes in CHR-individuals is an important objective.

We expect that the YouR-study will provide novel data to address these questions as well yield biomarkers that allow prediction of mental health outcomes in CHR-individuals. Specifically, we expect for the first-time a comprehensive characterization of the role of neural oscillations in emerging psychosis and its relationship to changes in E/I-balance parameters. While both rhythmic activity and alterations in GABA/Glutamatergic neurotransmission have been implicated in circuit abnormalities and cognitive deficits in established ScZ [15], their role in the early stages of psychosis is less clear. Accordingly, these data could inform novel approaches towards the atrisk mental state paradigm as well as allow the search for targeted approaches to correct abnormalities in circuit dysfunctions which could eventually lead to the prevention of severe mental health outcomes during the transition from adolescence to adulthood.

\begin{abstract}
Abbreviations
ACES: Adverse childhood experience scale; ASS: Auditory steady-state; BACS: Brief Assessment of Cognition in Schizophrenia Battery; BAPS: Beliefs About Paranoia Scale; BCSS: The Brief Core Schema Scale; BLIPs: Brief limited intermittent psychotic episodes; BS: Basic symptom; CAARMS: Comprehensive Assessment of At-Risk Mental State; CAMHS: Child and Adolescent Mental Health Services; CCNi: Centre for Cognitive Neuroimaging; CHR: Clinical high-risk criteria; CMHTs: Community Mental Health Teams; E/I: Excitatoryinhibition; ERFs: Event-related fields; ERPs: Event-related potentials; FE: Firstepisode; fMRI: Functional Magnetic Resonance Imaging; FROGS: Functional Remission in General Schizophrenia; GAF: Global Assessment of Functioning; GLM: Generalized Linear Model; GIx: Glutamate/Glutamine; I-PANASSF: International Positive and Negative Affect Schedule, short-form; MEG: Magnetocencephalography; MEGA-PRESS: MEGA point-resolved Spectroscopy; MMNm: Magnetic mismatch negativity; MNE: Minimum Norm Estimates; MNI: Montreal Neurological Institute; MRI: Magnetic Resonance Imaging; MRS: Magnetic Resonance Spectroscopy; PAM: Psychosis Attachment Measure; PCA: Principal component analysis; PCMHTs: Primary Care Mental Health Teams; PennCNP: University of Pennsylvania Computerized Neuropsychological Testing Battery; RISC: Rust Inventory of Schizotypal Cognitions; ScZ: Schizophrenia; SIPS: Structured Interview for Prodromal Symptoms; SPI-A: Schizophrenia Proneness Instrument, Adult Version; SPM: Statistical Parametric Mapping; UHR: Ultra-high risk; YouR-Study: Youth Mental Health Risk and Resilience Study; DSM: Diagnostic and Statistical Manual of the American Psychiatric Association
\end{abstract}

\section{Acknowledgements}

We thank Tineke Grent-T-Jong for useful comments on an earlier version of the manuscript.

\section{Funding}

This study was supported by the project MR/L011689/1 from the Medical Research Council (MRC).

\section{Availability of data and material}

The datasets used and/or analysed during the current study are available from the corresponding author on reasonable request. 


\section{Authors' contributions}

PJU wrote the first draft of the manuscript, planned the study, made substantial contribution to the development of the study protocol, as well as overall management of the study. AIG contributed to the application for funding, made substantial contribution to the design of the study and protocol, and critically read the manuscript. RG made substantial contribution to the design of the study and protocol, and critically read the manuscript. JG contributed to the application for funding, made substantial contribution to the design of the study and protocol, the statistical analysis plan, and critically read the manuscript. SML contributed to the application for funding, made substantial contribution to the design of the study and protocol, and critically read the manuscript. MS contributed to the application for funding, made substantial contribution to the design of the study and protocol, and critically read the manuscript. All authors read and approved the final manuscript.

\section{Competing interests}

Peter Uhlhaas has received research funding from Lilly and Lundbeck Pharmaceuticals.

\section{Consent for publication}

N/A

\section{Ethics approval and consent to participate}

The study is approved by the West of Scotland Research Ethics Service (14/WS/0099). Participation in the YouR-study will be based on written informed consent. The YouR-study comprises of 2 phases: initial screening and a further assessment phases. Consent will be taken following the initial screening phase for those meeting the initial screening criteria.

\section{Author details}

${ }^{1}$ Institute for Neuroscience and Psychology, University of Glasgow, 58 Hillhead Street, Glasgow, Scotland. ${ }^{2}$ Mental Health and Wellbeing, Institute of Health and Wellbeing, University of Glasgow, Glasgow, Scotland. ${ }^{3}$ Department of Psychiatry, University of Edinburgh, Edinburgh, Scotland. ${ }^{4}$ Department of Clinical Psychology, University Edinburgh, Edinburgh, Scotland.

Received: 9 January 2017 Accepted: 13 January 2017

\section{Published online: 26 January 2017}

\section{References}

1. Paus T, Keshavan M, Giedd JN. Why do many psychiatric disorders emerge during adolescence? Nat Rev Neurosci. 2008;9(12):947-57.

2. Kessler RC, et al. Lifetime prevalence and age-of-onset distributions of DSM-IV disorders in the National Comorbidity Survey Replication. Arch Gen Psychiatry. 2005;62(6):593-602.

3. Lee FS, et al. Mental health. Adolescent mental health-opportunity and obligation. Science. 2014;346(6209):547-9.

4. Fusar-Poli $P$, et al. The psychosis high-risk state: a comprehensive state-ofthe-art review. JAMA Psychiat. 2013;70(1):107-20.

5. Klosterkotter J, et al. Diagnosing schizophrenia in the initial prodromal phase. Arch Gen Psychiatry. 2001;58(2):158-64.

6. Yung AR, et al. Mapping the onset of psychosis: the Comprehensive Assessment of At-Risk Mental States. Aust N Z J Psychiatry. 2005;39(11-12): 964-71

7. Miller TJ, et al. Prodromal assessment with the structured interview for prodromal syndromes and the scale of prodromal symptoms: predictive validity, interrater reliability, and training to reliability. Schizophr Bull. 2003; 29(4):703-15.

8. Schultze-Lutter $F$, et al. Basic symptoms and ultrahigh risk criteria: symptom development in the initial prodromal state. Schizophr Bull. 2010;36(1):182-91.

9. Schmidt SJ, et al. EPA guidance on the early intervention in clinical high risk states of psychoses. Eur Psychiatry. 2015;30(3):388-404.

10. Schultze-Lutter F, Klosterkotter J, Ruhrmann S. Improving the clinical prediction of psychosis by combining ultra-high risk criteria and cognitive basic symptoms. Schizophr Res. 2014;154(1-3):100-6.

11. Fusar-Poli $P$, et al. At risk or not at risk? A meta-analysis of the prognostic accuracy of psychometric interviews for psychosis prediction. World Psychiatry. 2015;14(3):322-32.
12. Bechdolf $\mathrm{A}$, et al. Experience of trauma and conversion to psychosis in an ultra-high-risk (prodromal) group. Acta Psychiatr Scand. 2010;121(5):377-84.

13. Thompson A, et al. Borderline personality features and development of psychosis in an 'ultra high risk' (UHR) population: a case control study. Early Interv Psychiatry. 2012;6(3):247-55.

14. Yung $A R$, et al. Whither the attenuated psychosis syndrome? Schizophr Bull. 2012;38(6):1130-4

15. Uhlhaas PJ, Singer W. Neuronal dynamics and neuropsychiatric disorders: toward a translational paradigm for dysfunctional large-scale networks. Neuron. 2012;75(6):963-80.

16. Uhlhaas PJ, Singer W. Neural synchrony in brain disorders: relevance for cognitive dysfunctions and pathophysiology. Neuron. 2006;52(1):155-68

17. Fries P. Neuronal gamma-band synchronization as a fundamental process in cortical computation. Annu Rev Neurosci. 2009;32:209-24.

18. Jensen O, Kaiser J, Lachaux JP. Human gamma-frequency oscillations associated with attention and memory. Trends Neurosci. 2007;30(7):317-24.

19. Wang XJ. Neurophysiological and computational principles of cortical rhythms in cognition. Physiol Rev. 2010;90(3):1195-268.

20. Sohal VS, et al. Parvalbumin neurons and gamma rhythms enhance cortical circuit performance. Nature. 2009;459(7247):698-702.

21. Fuchs EC, et al. Genetically altered AMPA-type glutamate receptor kinetics in interneurons disrupt long-range synchrony of gamma oscillation. Proc Natl Acad Sci U S A. 2001;98(6):3571-6.

22. Kegeles LS, et al. Elevated prefrontal cortex gamma-aminobutyric acid and glutamate-glutamine levels in schizophrenia measured in vivo with proton magnetic resonance spectroscopy. 2012. Arch Gen Psychiatry.

23. Kring AM, Elis O. Emotion deficits in people with schizophrenia. 2012. Annu Rev Clin Psychol.

24. Isvoranu AM, et al. A network approach to environmental impact in psychotic disorder: brief theoretical framework. Schizophr Bull. 2016:42(4): 870-3.

25. Kimhy $\mathrm{D}$, et al. The impact of emotion awareness and regulation on socia functioning in individuals at clinical high risk for psychosis. Psychol Med. 2016:46(14):2907-18

26. Ising HK, et al. The validity of the 16-item version of the Prodromal Questionnaire (PQ-16) to screen for ultra high risk of developing psychosis in the general help-seeking population. Schizophr Bull. 2012;38(6):1288-96.

27. Roux F, et al. Gamma-band activity in human prefrontal cortex codes for the number of relevant items maintained in working memory. J Neurosci. 2012:32(36):12411-20.

28. Sheehan DV, et al. The Mini-International Neuropsychiatric Interview (M.I.N.I. ): the development and validation of a structured diagnostic psychiatric interview for DSM-IV and ICD-10. J Clin Psychiatry. 1998;59 Suppl 20:22-33. quiz 34-57.

29. Rabinowitz J, et al. The premorbid adjustment scale structured interview (PAS-SI): preliminary findings. Schizophr Res. 2007;90(1-3):255-7.

30. Cornblatt BA, et al. Preliminary findings for two new measures of social and role functioning in the prodromal phase of schizophrenia. Schizophr Bull. 2007:33(3):688-702

31. Tarbox SI, et al. Premorbid functional development and conversion to psychosis in clinical high-risk youths. Dev Psychopathol. 2013;25(4 Pt 1): 1171-86.

32. Salokangas RK, et al. Short-term functional outcome and premorbid adjustment in clinical high-risk patients. Results of the EPOS project. Eur Psychiatry. 2014;29(6):371-80.

33. Cannon TD, et al. An individualized risk calculator for research in prodromal psychosis. Am J Psychiatry. 2016;173(10):980-8.

34. Fusar-Poli $P$, et al. Cognitive functioning in prodromal psychosis: a metaanalysis. Arch Gen Psychiatry. 2012;69(6):562-71.

35. Seidman $L$, et al. Association of neurocognition with transition to psychosis: baseline functioning in the second phase of the North American prodrome longitudinal study. JAMA Psychiat. 2016;73(12):1239-48.

36. Keefe RS, et al. The Brief Assessment of Cognition in Schizophrenia: reliability, sensitivity, and comparison with a standard neurocognitive battery. Schizophr Res. 2004;68(2-3):283-97.

37. Moore TM, et al. Psychometric properties of the penn computerized neurocognitive battery. 2014. Neuropsychology.

38. Oldfield RC. The assessment and analysis of handedness: the Edinburgh inventory. Neuropsychologia. 1971;9(1):97-113.

39. Nelson HE The National Adult Reading Test (NART): test manual. Windsor: NFER-Nelson; 1982. 
40. Gumley Al, et al. The development and validation of the Beliefs about Paranoia Scale (Short Form). Behav Cogn Psychother. 2011;39(1):35-53.

41. Fowler D, et al. The Brief Core Schema Scales (BCSS): psychometric properties and associations with paranoia and grandiosity in non-clinical and psychosis samples. Psychol Med. 2006;36(6):749-59.

42. Korver-Nieberg $\mathrm{N}$, et al. Adult attachment and psychotic phenomenology in clinical and non-clinical samples: a systematic review. Psychol Psychother. 2014;87(2):127-54.

43. Felitti VJ, et al. Relationship of childhood abuse and household dysfunction to many of the leading causes of death in adults. The Adverse Childhood Experiences (ACE) Study. Am J Prev Med. 1998;14(4):245-58.

44. Rust J. The Rust Inventory of Schizotypal Cognitions (RISC). Schizophr Bull. 1988;14(2):317-22.

45. Barkham M, Hardy GE, Startup M. The IIP-32: a short version of the Inventory of Interpersonal Problems. Br J Clin Psychol. 1996:35(Pt 1):21-35.

46. Power MJ, Champion LA, Aris SJ. The development of a measure of social support: the Significant Others (SOS) Scale. Br J Clin Psychol. 1988;27(Pt 4): 349-58.

47. Thompon ER. Development and validation of an internationally reliable short-form of the Positive and Negative Affect Schedule (PANAS). J Cross Cult Psychol. 2007:38(2):227-42.

48. Mattick RP, Clarke JC. Development and validation of measures of social phobia scrutiny fear and social interaction anxiety. Behav Res Ther. 1998; 36(4):455-70.

49. Llorca PM, et al. The "Functional Remission of General Schizophrenia" (FROGS) scale: development and validation of a new questionnaire. Schizophr Res. 2009;113(2-3):218-25.

50. Oostenveld R, et al. FieldTrip: open source software for advanced analysis of MEG, EEG, and invasive electrophysiological data. Comput Intell Neurosci. 2011;2011:156869.

51. Gross J, et al. Dynamic imaging of coherent sources: studying neural interactions in the human brain. Proc Natl Acad Sci U S A. 2001;98(2):694-9.

52. Dale AM, et al. Dynamic statistical parametric mapping: combining fMRI and MEG for high-resolution imaging of cortical activity. Neuron. 2000;26(1): 55-67.

53. Baccala LA, Sameshima K. Partial directed coherence: a new concept in neural structure determination. Biol Cybern. 2001;84(6):463-74.

54. Ploner $\mathrm{M}$, et al. Functional integration within the human pain system as revealed by Granger causality. Hum Brain Mapp. 2009;30(12):4025-32.

55. Stam CJ, Nolte G, Daffertshofer A. Phase lag index: assessment of functional connectivity from multi channel EEG and MEG with diminished bias from common sources. Hum Brain Mapp. 2007;28(11):1178-93.

56. Hoogenboom N, et al. Localizing human visual gamma-band activity in frequency, time and space. Neuroimage. 2006;29(3):764-73.

57. Tan HM, Gross J, Uhlhaas PJ. MEG sensor and source measures of visually induced gamma-band oscillations are highly reliable. 2016. Neuroimage.

58. Hoogenboom N, et al. Visually induced gamma-band activity predicts speed of change detection in humans. Neuroimage. 2010;51(3):1162-7.

59. Womelsdorf T, et al. Gamma-band synchronization in visual cortex predicts speed of change detection. Nature. 2006;439(7077):733-6.

60. Grent t'-Jong, T.e.a., MEG-measured visually induced gamma-band oscillations in chronic schizophrenia: evidence for impaired generation of rhythmic activity in ventral stream regions. Schizophr Res. 2016. in press.

61. Kwon JS, et al. Gamma frequency-range abnormalities to auditory stimulation in schizophrenia. Arch Gen Psychiatry. 1999;56(11):1001-5.

62. Thune $\mathrm{H}$, Recasens $\mathrm{M}$, Uhlhaas PJ. The $40-\mathrm{Hz}$ auditory steady-state response in patients with schizophrenia: a meta-analysis. JAMA Psychiat. 2016;73(11): 1145-53.

63. Brown $\mathrm{H}$, et al. Active inference, sensory attenuation and illusions. Cogn Process. 2013;14(4):411-27.

64. Perez VB, et al. Auditory cortex responsiveness during talking and listening: early illness schizophrenia and patients at clinical high-risk for psychosis. Schizophr Bull. 2012;38(6):1216-24.

65. Naatanen $\mathrm{R}$, et al. The mismatch negativity $(\mathrm{MMN})$ in basic research of central auditory processing: a review. Clin Neurophysiol. 2007;118(12): 2544-90.

66. Erickson MA, Ruffle A, Gold JM. A meta-analysis of mismatch negativity in schizophrenia: from clinical risk to disease specificity and progression. Biol Psychiatry. 2016;79(12):980-7.

67. Bodatsch $\mathrm{M}$, et al. Prediction of psychosis by mismatch negativity. Bio Psychiatry. 2011;69(10):959-66.
68. Grutzner $\mathrm{C}$, et al. Deficits in high- $(>60 \mathrm{~Hz})$ gamma-band oscillations during visual processing in schizophrenia. Front Hum Neurosci. 2013;7:88.

69. Sun $L$, et al. Impaired gamma-band activity during perceptual organization in adults with autism spectrum disorders: evidence for dysfunctiona network activity in frontal-posterior cortices. J Neurosci. 2012;32(28): 9563-73.

70. Smieskova R, et al. Neuroimaging predictors of transition to psychosis-a systematic review and meta-analysis. Neurosci Biobehav Rev. 2010;34(8): 1207-22.

71. Atkinson RJ, Michie PT, Schall U. Duration mismatch negativity and P3a in first-episode psychosis and individuals at ultra-high risk of psychosis. Biol Psychiatry. 2012;71(2):98-104.

72. Koutsouleris $\mathrm{N}$, et al. Use of neuroanatomical pattern classification to identify subjects in at-risk mental states of psychosis and predict disease transition. Arch Gen Psychiatry. 2009;66(7):700-12.

\section{Submit your next manuscript to BioMed Central and we will help you at every step:}

- We accept pre-submission inquiries

- Our selector tool helps you to find the most relevant journal

- We provide round the clock customer support

- Convenient online submission

- Thorough peer review

- Inclusion in PubMed and all major indexing services

- Maximum visibility for your research

Submit your manuscript at www.biomedcentral.com/submit
Biomed Central 